\title{
Some inequalities based on a general quantum difference operator
}

\author{
Alaa E Hamza ${ }^{1}$ and Enas M Shehata ${ }^{2 *}$
}

\section{"Correspondence:}

enasmohyi@yahoo.com

2Department of Mathematics,

Faculty of Science, Menoufia

University, Shibin El-Kom, Egypt

Full list of author information is

available at the end of the article

\begin{abstract}
In this paper, some integral inequalities based on the general quantum difference operator $D_{\beta}$ are deduced. Here, $D_{\beta}$ is defined by $D_{\beta} f(t)=(f(\beta(t))-f(t)) /(\beta(t)-t)$, where $\beta$ is a strictly increasing continuous function, defined on an interval $I \subseteq \mathbb{R}$, that has one fixed point $s_{0} \in I$. The $\beta$-Hölder and $\beta$-Minkowski inequalities are proved. Also, the $\beta$-Gronwall, $\beta$-Bernoulli, and some related inequalities are shown. Finally, the $\beta$-Lyapunov inequality is established.
\end{abstract}

MSC: 39A10; 39A13; 39A70; 47B39

Keywords: quantum difference operator; quantum calculus; Hölder inequality; Minkowski inequality; Gronwall inequality; Bernoulli inequality; Lyapunov inequality

\section{Introduction}

Quantum difference operators have an interest role due to their applications; see, e.g., [1-7] and references cited therein. A calculus based on a quantum difference operator is usually known as calculus without limits. It substitutes the classical derivative by a difference operator, which allows one to deal with sets of nondifferentiable functions. In [8] Hahn introduced his difference operator, as a tool for constructing families of orthogonal polynomials, which is defined by

$$
D_{q, \omega} f(t)=\frac{f(q t+\omega)-f(t)}{t(q-1)+\omega}, \quad t \neq \omega_{0},
$$

where $q \in(0,1), \omega>0$ are fixed and $\omega_{0}=\frac{\omega}{1-q}$. The derivative at $t=\omega_{0}$ is defined to be $f^{\prime}\left(\omega_{0}\right)$ whenever it exists. In [1], its inverse operator was constructed and a rigorous analysis of the calculus associated to $D_{q, \omega}$ was given. See also [9-11]. The Hahn quantum difference operator unifies two well-known difference operators. The first one is the Jackson $q$-difference operator, which is defined by

$$
D_{q} f(t)=\frac{f(q t)-f(t)}{t(q-1)}, \quad t \neq 0
$$

where $q$ is a fixed number, $q \in(0,1)$. The function $f$ is defined on a $q$-geometric set $\mathbb{A} \subseteq \mathbb{R}$ (or $\mathbb{C}$ ) such that whenever $t \in \mathbb{A}, q t \in \mathbb{A}$. At $t=0, D_{q} f(t)=f^{\prime}(0)$. The second difference operator is the difference operator $D_{\omega}$, which is defined by

(c) 2015 Hamza and Shehata; licensee Springer. This is an Open Access article distributed under the terms of the Creative Commons Attribution License (http://creativecommons.org/licenses/by/4.0), which permits unrestricted use, distribution, and reproduction in any medium, provided the original work is properly credited. 


$$
D_{\omega} f(t)=\frac{f(t+\omega)-f(t)}{\omega}, \quad t \in \mathbb{R}
$$

where the fixed number $\omega \neq 0$.

We refer the reader to the interesting books of Annaby and Mansour [2], Kac and Cheung [5], and Bangerezako [12].

In [13] we introduced a general quantum difference operator $D_{\beta}$ defined by

$$
D_{\beta} f(t)=\frac{f(\beta(t))-f(t)}{\beta(t)-t},
$$

for every $t$ with $\beta(t) \neq t$, where $f$ is an arbitrary function defined, in general, on a $\beta$ geometric set $I \subseteq \mathbb{R}$, for which $\beta(t) \in I, t \in I$, and $\beta: I \rightarrow I$ is a strictly increasing continuous function. For a fixed point $s_{0}$ of $\beta$, the $\beta$-derivative $D_{\beta} f(t)$ at $t=s_{0}$ is defined to be $f^{\prime}\left(s_{0}\right)$, whenever $f$ is differentiable at $t=s_{0}$ in the usual sense. The function $\beta$ has many forms due to its properties. It may be linear or nonlinear. Accordingly, it has no fixed points or has at least one. Every choice of the function $\beta$ gives a new difference operator. Thus, we can obtain a wide class of difference operators with the corresponding quantum calculi. We have a space of forms of the function $\beta$ which may be classified into classes according to the number of fixed points beside the directions of the sequences $\left\{\beta^{k}(t)\right\}_{k \in \mathbb{N}_{0}}$ towards and outwards these points. The $\beta$-difference operator yields the Hahn difference operator when $\beta(t)=q t+\omega, \omega>0$, and the Jackson $q$-difference operator when $\beta(t)=q t$, $q \in(0,1)$. In these cases $\beta$ is linear and has one fixed point. Also, the forward difference operator $D_{\omega}$ with the linear form of $\beta(t)=t+\omega, \omega>0$, has no fixed points. Consequently, the corresponding Hahn calculus, $q$-calculus, and $\omega$-calculus, respectively, are particular cases of the $\beta$-calculus. In [13] we considered the class of our function $\beta$ which has one fixed point $s_{0} \in I$ and satisfies the following condition:

$$
\left(t-s_{0}\right)(\beta(t)-t) \leq 0 \quad \text { for all } t \in I \text {. }
$$

This paper is devoted to deducing some basic integral inequalities based on the $\beta$ difference operator, when $\beta$ has one fixed point $s_{0} \in I$ and satisfies the same condition. The paper is organized as follows. In Section 2, we exhibit the results that we need from [13], concerning the calculus based on the $\beta$-difference operator. Section 3 contains our main results. We prove the $\beta$-Hölder, $\beta$-Minkowski, $\beta$-Gronwall, and $\beta$-Bernoulli inequalities and some related ones. Finally, we show the $\beta$-Lyapunov inequality. These inequalities are very important in establishing the theory of $\beta$-difference equations associated with the quantum difference operator $D_{\beta}$.

Throughout this paper $I$ is an interval of $\mathbb{R}$ containing the fixed point $s_{0}$ of $\beta$ and $\mathbb{X}$ is a Banach space with norm $\|\cdot\|$.

\section{Preliminaries}

In this section we present some needed results from [13] concerning the calculus associated with $D_{\beta}$. Here, we consider the class of the function $\beta$ when it has one fixed point $s_{0} \in I$ and satisfies the following condition:

$$
\left(t-s_{0}\right)(\beta(t)-t) \leq 0 \quad \text { for all } t \in I \text {. }
$$


Lemma 2.1 The following statements are true:

(i) The sequence of functions $\left\{\beta^{k}(t)\right\}_{k \in \mathbb{N}_{0}}$ converges uniformly to the constant function $\hat{\beta}(t):=s_{0}$ on every compact interval $\subseteq \subseteq$ I containing $s_{0}$.

(ii) The series $\sum_{k=0}^{\infty}\left|\beta^{k}(t)-\beta^{k+1}(t)\right|$ is uniformly convergent to $\left|t-s_{0}\right|$ on every compact interval $J \subseteq I$ containing $s_{0}$.

Lemma 2.2 If $f: I \rightarrow \mathbb{X}$ is continuous at $s_{0}$, then the sequence $\left\{f\left(\beta^{k}(t)\right)\right\}_{k \in \mathbb{N}_{0}}$ converges uniformly to $f\left(s_{0}\right)$ on every compact interval $J \subseteq$ I containing $s_{0}$.

Theorem 2.3 If $f: I \rightarrow \mathbb{X}$ is continuous at $s_{0}$, then the series $\sum_{k=0}^{\infty} \|\left(\beta^{k}(t)-\beta^{k+1}(t)\right) \times$ $f\left(\beta^{k}(t)\right) \|$ is uniformly convergent on every compact interval $J \subseteq I$ containing $s_{0}$.

Definition 2.4 For a function $f: I \rightarrow \mathbb{X}$, we define the $\beta$-difference operator of $f$ as

$$
D_{\beta} f(t)= \begin{cases}\frac{f(\beta(t))-f(t)}{\beta(t)-t}, & t \neq s_{0}, \\ f^{\prime}\left(s_{0}\right), & t=s_{0},\end{cases}
$$

provided that the ordinary derivative $f^{\prime}$ exists at $s_{0}$. In this case, we say that $D_{\beta} f(t)$ is the $\beta$-derivative of $f$ at $t$. We say that $f$ is $\beta$-differentiable on $I$ if $f^{\prime}\left(s_{0}\right)$ exists.

Theorem 2.5 Assume that $f: I \rightarrow \mathbb{X}$ and $g: I \rightarrow \mathbb{R}$ are $\beta$-differentiable at $t \in I$. Then:

(i) The product $f g: I \rightarrow \mathbb{X}$ is $\beta$-differentiable at $t$ and

$$
\begin{aligned}
D_{\beta}(f g)(t) & =\left(D_{\beta} f(t)\right) g(t)+f(\beta(t)) D_{\beta} g(t) \\
& =\left(D_{\beta} f(t)\right) g(\beta(t))+f(t) D_{\beta} g(t) .
\end{aligned}
$$

(ii) $\mathrm{f} / \mathrm{g}$ is $\beta$-differentiable at $t$ and

$$
D_{\beta}(f / g)(t)=\frac{\left(D_{\beta} f(t)\right) g(t)-f(t) D_{\beta} g(t)}{g(t) g(\beta(t))},
$$

provided that $g(t) g(\beta(t)) \neq 0$.

Lemma 2.6 Let $f: I \rightarrow \mathbb{X}$ be $\beta$-differentiable and $D_{\beta} f(t)=0$ for all $t \in I$, then $f(t)=f\left(s_{0}\right)$ for all $t \in I$.

Corollary 2.7 Suppose that $f, g: I \rightarrow \mathbb{X}$ are $\beta$-differentiable on I. If $D_{\beta} f(t)=D_{\beta} g(t)$ for all $t \in I$, then

$$
f(t)-g(t)=f\left(s_{0}\right)-g\left(s_{0}\right) \quad \text { for all } t \in I .
$$

Theorem 2.8 Assume $f: I \rightarrow \mathbb{X}$ is continuous at $s_{0}$. Then the function $F$ defined by

$$
F(t)=\sum_{k=0}^{\infty}\left(\beta^{k}(t)-\beta^{k+1}(t)\right) f\left(\beta^{k}(t)\right), \quad t \in I,
$$

is a $\beta$-antiderivative off with $F\left(s_{0}\right)=0$. Conversely, a $\beta$-antiderivative $F$ off vanishing at $s_{0}$ is given by (2.1). 
Definition 2.9 Let $f: I \rightarrow \mathbb{X}$ and $a, b \in I$. We define the $\beta$-integral of $f$ from $a$ to $b$ by

$$
\int_{a}^{b} f(t) d_{\beta} t=\int_{s_{0}}^{b} f(t) d_{\beta} t-\int_{s_{0}}^{a} f(t) d_{\beta} t
$$

where

$$
\int_{s_{0}}^{x} f(t) d_{\beta} t=\sum_{k=0}^{\infty}\left(\beta^{k}(x)-\beta^{k+1}(x)\right) f\left(\beta^{k}(x)\right), \quad x \in I,
$$

provided that the series converges at $x=a$ and $x=b . f$ is called $\beta$-integrable on $I$ if the series converges at $a, b$ for all $a, b \in I$. Clearly, if $f$ is continuous at $s_{0} \in I$, then $f$ is $\beta$ integrable on $I$.

If $\beta(t)=q t$ and $\beta(t)=q t+\omega, q \in(0,1), \omega>0$, then (2.2) and (2.3) reduce to the Jackson $q$-integral and Hahn integral, respectively; see $[1,5,9,12,14]$.

Theorem 2.10 Let $f: I \rightarrow \mathbb{X}$ be continuous at $s_{0}$. Define the function

$$
F(x)=\int_{s_{0}}^{x} f(t) d_{\beta} t, \quad x \in I .
$$

Then $F$ is continuous at $s_{0}, D_{\beta} F(x)$ exists for all $x \in I$ and $D_{\beta} F(x)=f(x)$.

Theorem 2.11 Iff $: I \rightarrow \mathbb{X}$ is $\beta$-differentiable on $I$, then

$$
\int_{a}^{b}\left(D_{\beta} f\right)(t) d_{\beta} t=f(b)-f(a), \quad \text { for all } a, b \in I
$$

Theorem 2.12 Assume $f$, $g$ are $\beta$-differentiable functions on $I$ and $D_{\beta} f, D_{\beta} g$ both continuous at $s_{0}$. Then

$$
\int_{a}^{b} f(t) D_{\beta} g(t) d_{\beta} t=f(b) g(b)-f(a) g(a)-\int_{a}^{b}\left(D_{\beta} f(t)\right) g(\beta(t)) d_{\beta} t, \quad a, b \in I .
$$

Here at least one of the functions $f$ and $g$ is a real valued function.

Definition 2.13 Let $s_{0} \in[a, b] \subseteq I$. We define the $\beta$-interval by

$$
[a, b]_{\beta}=\left\{\beta^{k}(a) ; k \in \mathbb{N}_{0}\right\} \cup\left\{\beta^{k}(b) ; k \in \mathbb{N}_{0}\right\} \cup\left\{s_{0}\right\} .
$$

For any point $c \in I$, we denote by

$$
[c]_{\beta}=\left\{\beta^{k}(c) ; k \in \mathbb{N}_{0}\right\} \cup\left\{s_{0}\right\} .
$$

Finally, for $A \subseteq \mathbb{R}$, we denote by

$$
A^{*}=A \backslash\left\{s_{0}\right\} .
$$


Let $D$ denote the set of all real valued functions defined on $[c, d]_{\beta}$ and continuous at $s_{0}$, where $c, d \in I$ and $c<d$.

Lemma 2.14 Let $f \in D$. Then $\int_{c}^{d} f(t) h(\beta(t)) d_{\beta} t=0$ for all functions $h \in D$ with $h(c)=$ $h(d)=0$ if and only iff $(t)=0$ for all $t \in[c, d]_{\beta}$.

Lemma 2.15 Let $f: I \rightarrow \mathbb{X}, g: I \rightarrow \mathbb{R}$ be $\beta$-integrable functions on $I$. If

$$
\|f(t)\| \leq g(t) \quad \text { for all } t \in[a, b]_{\beta}, a, b \in I, a \leq b,
$$

then for $x, y \in[a, b]_{\beta}, x<s_{0}<y$, we have

$$
\begin{aligned}
\left\|\int_{s_{0}}^{y} f(t) d_{\beta} t\right\| & \leq \int_{s_{0}}^{y} g(t) d_{\beta} t, \\
\left\|\int_{s_{0}}^{x} f(t) d_{\beta} t\right\| & \leq \int_{x}^{s_{0}} g(t) d_{\beta} t,
\end{aligned}
$$

and

$$
\left\|\int_{x}^{y} f(t) d_{\beta} t\right\| \leq \int_{x}^{y} g(t) d_{\beta} t
$$

Consequently, if $g(t) \geq 0$ for all $t \in[a, b]_{\beta}$, then the inequalities $\int_{s_{0}}^{y} g(t) d_{\beta} t \geq 0$ and $\int_{x}^{y} g(t) d_{\beta} t \geq 0$ hold for all $x, y \in[a, b]_{\beta}, a, b \in I, a \leq b$.

Lemma 2.16 Let $f: I \rightarrow \mathbb{X}$ and $g: I \rightarrow \mathbb{R}$ be $\beta$-differentiable on I. If

$$
\left\|D_{\beta} f(t)\right\| \leq D_{\beta} g(t), \quad t \in[a, b]_{\beta}, a, b \in I, a \leq b,
$$

then

$$
\|f(y)-f(x)\| \leq g(y)-g(x)
$$

for every $x, y \in[a, b]_{\beta}, x<s_{0}<y$.

Definition 2.17 ( $\beta$-exponential functions) Assume that $p: I \rightarrow \mathbb{C}$ is a continuous function at $s_{0}$. We define the $\beta$-exponential functions $e_{p, \beta}(t)$ and $E_{p, \beta}(t)$ by

$$
e_{p, \beta}(t)=\frac{1}{\prod_{k=0}^{\infty}\left[1-p\left(\beta^{k}(t)\right)\left(\beta^{k}(t)-\beta^{k+1}(t)\right)\right]}
$$

and

$$
E_{p, \beta}(t)=\prod_{k=0}^{\infty}\left[1+p\left(\beta^{k}(t)\right)\left(\beta^{k}(t)-\beta^{k+1}(t)\right)\right],
$$

whenever both infinite products are convergent to a non-zero number for every $t \in I$. Clearly, we have

$$
e_{p, \beta}(t)=\frac{1}{E_{-p, \beta}(t)} .
$$


Theorem 2.18 The $\beta$-exponential functions $e_{p, \beta}(t)$ and $E_{p, \beta}(t)$ are the unique solutions of the first order $\beta$-difference equations

$$
D_{\beta} y(t)=p(t) y(t), \quad y\left(s_{0}\right)=1,
$$

and

$$
D_{\beta} y(t)=p(t) y(\beta(t)), \quad y\left(s_{0}\right)=1,
$$

respectively.

\section{Main results}

\section{1 $\beta$-Hölder and $\beta$-Minkowski inequalities}

Inspired in the work by Agarwal et al. [15], we present the $\beta$-Hölder, $\beta$-Cauchy-Schwarz, and $\beta$-Minkowski inequalities.

Definition 3.1 Let $p \geq 1$ and $a, b \in I \subseteq \mathbb{R}, a \leq b$. We denote by $L_{\beta}^{p}[a, b]_{\beta}$ the space of all functions $f:[a, b]_{\beta} \rightarrow \mathbb{R}$ such that

$$
\sup \left\{\int_{x}^{y}|f(t)|^{p} d_{\beta} t: x, y \in[a, b]_{\beta}, s_{0} \in[x, y]\right\}<\infty .
$$

Theorem 3.2 ( $\beta$-Hölder inequality) Let $f \in L_{\beta}^{p}[a, b]_{\beta}$ and $g \in L_{\beta}^{q}[a, b]_{\beta}$. Then $|f g| \in$ $L_{\beta}^{1}[a, b]_{\beta}$ and

$$
\int_{x}^{y}|f(t) g(t)| d_{\beta} t \leq\left(\int_{x}^{y}|f(t)|^{p} d_{\beta} t\right)^{1 / p}\left(\int_{x}^{y}|g(t)|^{q} d_{\beta} t\right)^{1 / q},
$$

where $x, y \in[a, b]_{\beta}, s_{0} \in[x, y]$, and $p>1, q=p /(p-1)$. The equality holds if $|f(t)|^{p} /|g(t)|^{q}$ is constant.

Proof For $\alpha, \gamma \in[0, \infty)$, we have

$$
\alpha^{1 / p} \gamma^{1 / q} \leq \frac{\alpha}{p}+\frac{\gamma}{q}
$$

Let $\alpha(t)=\frac{|f(t)|^{p}}{\left.\int_{x}^{y} f(t)\right|^{p} d_{\beta} t}$ and $\gamma(t)=\frac{|g(t)|^{q}}{\int_{x}^{y}|g(t)|^{q} d_{\beta} t}$, with

$$
\left(\int_{x}^{y}|f(t)|^{p} d_{\beta} t\right)\left(\int_{x}^{y}|g(t)|^{q} d_{\beta} t\right) \neq 0
$$

Substituting in (3.2) and applying Lemma 2.15, we get

$$
\begin{aligned}
\int_{x}^{y} \frac{|f(t)|}{\left(\int_{x}^{y}|f(t)|^{p} d_{\beta} t\right)^{1 / p}} \frac{|g(t)|}{\left(\int_{x}^{y}|g(t)|^{q} d_{\beta} t\right)^{1 / q}} d_{\beta} t \leq & \frac{1}{p} \int_{x}^{y} \frac{|f(t)|^{p}}{\int_{x}^{y}|f(t)|^{p} d_{\beta} t} d_{\beta} t \\
& +\frac{1}{q} \int_{x}^{y} \frac{|g(t)|^{q}}{\int_{x}^{y}|g(t)|^{q} d_{\beta} t} d_{\beta} t \\
& =\frac{1}{p}+\frac{1}{q}=1 .
\end{aligned}
$$


Then

$$
\int_{x}^{y}|f(t) g(t)| d_{\beta} t \leq\left(\int_{x}^{y}|f(t)|^{p} d_{\beta} t\right)^{1 / p}\left(\int_{x}^{y}|g(t)|^{q} d_{\beta} t\right)^{1 / q} .
$$

It is obvious that if $|f(t)|^{p} /|g(t)|^{q}$ is constant, the equality holds.

Actually, if we put $p=q=2$ in the $\beta$-Hölder inequality we get the $\beta$-Cauchy-Schwarz inequality.

Corollary 3.3 ( $\beta$-Cauchy-Schwarz inequality) Let $f, g \in L_{\beta}^{2}[a, b]_{\beta}$. Then $|f g| \in L_{\beta}^{1}[a, b]_{\beta}$ and

$$
\int_{x}^{y}|f(t) g(t)| d_{\beta} t \leq \sqrt{\left(\int_{x}^{y}|f(t)|^{2} d_{\beta} t\right)\left(\int_{x}^{y}|g(t)|^{2} d_{\beta} t\right)},
$$

where $x, y \in[a, b]_{\beta}, s_{0} \in[x, y]$.

As in the classical Minkowski inequality, we can deduce the following result.

Theorem 3.4 ( $\beta$-Minkowski inequality) Let $1 \leq p<\infty$ and $a, b \in I, a \leq b$. Let $f, g \in$ $L_{\beta}^{p}[a, b]_{\beta}$. Then $|f+g| \in L_{\beta}^{p}[a, b]_{\beta}$ and

$$
\left(\int_{x}^{y}|(f+g)(t)|^{p} d_{\beta} t\right)^{1 / p} \leq\left(\int_{x}^{y}|f(t)|^{p} d_{\beta} t\right)^{1 / p}+\left(\int_{x}^{y}|g(t)|^{p} d_{\beta} t\right)^{1 / p},
$$

where $x, y \in[a, b]_{\beta}, s_{0} \in[x, y]$. The equality holds if $f(t) / g(t)$ is constant.

\section{2 $\beta$-Gronwall and $\beta$-Bernoulli inequalities}

Hamza and Ahmed, in [10], deduced the Hahn, Gronwall, and Bernoulli inequalities. In the following we present the corresponding $\beta$-version.

Lemma 3.5 Let $y, f, p$ are real valued functions defined on I and continuous at $s_{0}$. If

$$
D_{\beta} y(t) \leq p(t) y(t)+f(t) \quad \text { for all } t
$$

then

$$
y(t) \leq y\left(s_{0}\right) e_{p, \beta}(t)+e_{p, \beta}(t) \int_{s_{0}}^{t} f(\tau) E_{-p, \beta}(\beta(\tau)) d_{\beta} \tau .
$$

Proof We have

$$
\begin{aligned}
D_{\beta}\left(y(t) E_{-p, \beta}(t)\right) & =D_{\beta} y(t) E_{-p, \beta}(\beta(t))+y(t) D_{\beta} E_{-p, \beta}(t) \\
& =D_{\beta} y(t) E_{-p, \beta}(\beta(t))-p(t) y(t) E_{-p, \beta}(\beta(t)) \\
& =E_{-p, \beta}(\beta(t))\left(D_{\beta} y(t)-p(t) y(t)\right) \\
& \leq E_{-p, \beta}(\beta(t)) f(t) .
\end{aligned}
$$


Integrating both sides from $s_{0}$ to $t$ we get

$$
y(t) E_{-p, \beta}(t)-y\left(s_{0}\right) E_{-p, \beta}\left(s_{0}\right) \leq \int_{s_{0}}^{t} f(\tau) E_{-p, \beta}(\beta(\tau)) d_{\beta} \tau .
$$

In view of $E_{-p, \beta}\left(s_{0}\right)=1$ and $e_{p, \beta}(t)=\frac{1}{E_{-p, \beta}(t)}$, we conclude

$$
y(t) \leq y\left(s_{0}\right) e_{p, \beta}(t)+e_{p, \beta}(t) \int_{s_{0}}^{t} f(\tau) E_{-p, \beta}(\beta(\tau)) d_{\beta} \tau .
$$

Theorem 3.6 ( $\beta$-Gronwall inequality) Let $p \geq 0$ and $y, f, p$ be real valued continuous functions at $s_{0}$ defined on I. If

$$
y(t) \leq f(t)+\int_{s_{0}}^{t} y(\tau) p(\tau) d_{\beta} \tau
$$

then

$$
y(t) \leq f(t)+e_{p, \beta}(t) \int_{s_{0}}^{t} p(\tau) f(\tau) E_{-p, \beta}(\beta(\tau)) d_{\beta} \tau .
$$

Proof Define

$$
z(t)=\int_{s_{0}}^{t} y(\tau) p(\tau) d_{\beta} \tau
$$

Then $z\left(s_{0}\right)=0$ and $D_{\beta} z(t)=y(t) p(t)$. Therefore, inequality (3.6) yields

$$
y(t) \leq f(t)+z(t)
$$

and

$$
D_{\beta} z(t) \leq(f(t)+z(t)) p(t)
$$

By Lemma 3.5, we obtain

$$
z(t) \leq z\left(s_{0}\right) e_{p, \beta}(t)+e_{p, \beta}(t) \int_{s_{0}}^{t} p(\tau) f(\tau) E_{-p, \beta}(\beta(\tau)) d_{\beta} \tau .
$$

Inequality (3.10) implies

$$
y(t) \leq f(t)+e_{p, \beta}(t) \int_{s_{0}}^{t} p(\tau) f(\tau) E_{-p, \beta}(\beta(\tau)) d_{\beta} \tau .
$$

As a direct consequence, we obtain the following results.

Corollary 3.7 Let $p, y, f$ are continuous functions at $s_{0}$ and $p(t) \geq 0$. Then

$$
y(t) \leq \int_{s_{0}}^{t} y(\tau) p(\tau) d_{\beta} \tau, \quad \text { for all } t
$$


implies

$$
y(t) \leq 0 .
$$

Proof This is due to Theorem 3.6 with $f(t) \equiv 0$.

Corollary 3.8 Let $p(t) \geq 0$ and $\alpha \in \mathbb{R}$. Then

$$
y(t) \leq \alpha+\int_{s_{0}}^{t} y(\tau) p(\tau) d_{\beta} \tau, \quad \text { for all } t>s_{0}
$$

implies

$$
y(t) \leq \alpha e_{p, \beta}(t)
$$

Proof By the $\beta$-Gronwall inequality if we put $f(t)=\alpha$, then

$$
y(t) \leq \alpha+\int_{s_{0}}^{t} y(\tau) p(\tau) d_{\beta} \tau \text { for all } t .
$$

Consequently,

$$
\begin{aligned}
y(t) & \leq \alpha+e_{p, \beta}(t) \int_{s_{0}}^{t} \alpha p(\tau) E_{-p, \beta}(\beta(\tau)) d_{\beta} \tau \\
& =\alpha\left(1-e_{p, \beta}(t) \int_{s_{0}}^{t} D_{\beta} E_{-p, \beta}(\tau) d_{\beta} \tau\right) \\
& =\alpha\left(1-e_{p, \beta}(t)\left(E_{-p, \beta}(t)-E_{-p, \beta}\left(s_{0}\right)\right)\right. \\
& =\alpha-\alpha e_{p, \beta}(t) E_{-p, \beta}(t)+\alpha e_{p, \beta}(t) .
\end{aligned}
$$

Therefore, $y(t) \leq \alpha e_{p, \beta}(t)$.

Theorem 3.9 ( $\beta$-Bernoulli inequality) For $\alpha \in(0, \infty)$, the following inequality is true:

$$
e_{p, \beta}(t) \geq 1+\alpha\left(t-s_{0}\right), \quad t>s_{0} .
$$

Proof Suppose $y(t)=\alpha\left(t-s_{0}\right), t>s_{0}$. Then $D_{\beta} y(t)=\alpha$. We have $\alpha y(t)+\alpha=\alpha^{2}\left(t-s_{0}\right)+\alpha \geq$ $\alpha=D_{\beta} y(t)$, which implies that $D_{\beta} y(t) \leq \alpha y(t)+\alpha$. By Lemma 3.5 we get

$$
\begin{aligned}
y(t) & \leq y\left(s_{0}\right) e_{p, \beta}(t)+e_{p, \beta}(t) \int_{s_{0}}^{t} \alpha E_{-p, \beta}(\beta(\tau)) d_{\beta} \tau \\
& =e_{p, \beta}(t) \int_{s_{0}}^{t}-D_{\beta} E_{-p, \beta}(\tau) d_{\beta} \tau \\
& =-e_{p, \beta}(t)\left(E_{-p, \beta}(t)-1\right)=-1+e_{p, \beta}(t) .
\end{aligned}
$$

Therefore, $e_{p, \beta}(t) \geq 1+y(t)=1+\alpha\left(t-s_{0}\right), t>s_{0}$. 


\section{3 $\beta$-Lyapunov Inequality}

Lyapunov inequality has many applications in the theory of differential and difference equations. These applications include bounds for eigenvalues, stability criteria for periodic differential equations, and estimates for intervals of disconjugacy; see [16, 17]. In this section we introduce the Lyapunov inequality based on $D_{\beta}$.

Let $f: I \rightarrow[0, \infty)$ be a continuous function at $s_{0} \in I$. Consider the Sturm-Liouville $\beta$ difference equation

$$
D_{\beta}^{2} x(t)+f(t) x(\beta(t))=0, \quad t \in I
$$

Define the function $F$ by

$$
F(y)=\int_{a}^{b}\left[\left(D_{\beta} y(t)\right)^{2}-f(t)(y(\beta(t)))^{2}\right] d_{\beta} t .
$$

Lemma 3.10 Let $x$ be a nontrivial solution of the Sturm-Liouville $\beta$-difference equation (3.13). Then for every y belonging to the domain of $F$, the following equality holds:

$$
F(y)-F(x)-F(y-x)=2(y-x)(b) D_{\beta} x(b)-2(y-x)(a) D_{\beta} x(a) .
$$

Proof Simple calculations show that

$$
\begin{aligned}
F(y) & -F(x)-F(y-x) \\
= & \int_{a}^{b}\left\{\left(D_{\beta} y(t)\right)^{2}-f(t)(y(\beta(t)))^{2}-\left(D_{\beta} x(t)\right)^{2}+f(t)(x(\beta(t)))^{2}\right. \\
& \left.-\left(D_{\beta}(y-x)(t)\right)^{2}+f(t)((y-x)(\beta(t)))^{2}\right\} d_{\beta} t \\
= & 2 \int_{a}^{b}\left\{-\left(D_{\beta} x(t)\right)^{2}+f(t)(x(\beta(t)))^{2}+D_{\beta} y(t) D_{\beta} x(t)\right. \\
& -f(t) y(\beta(t)) x(\beta(t))\} d_{\beta} t \\
= & 2 \int_{a}^{b}\left\{D_{\beta} y(t) D_{\beta} x(t)+y(\beta(t)) D_{\beta}^{2} x(t)-\left(\left(D_{\beta} x(t)\right)^{2}\right.\right. \\
& \left.\left.+D_{\beta}^{2} x(t) x(\beta(t))\right)\right\} d_{\beta} t \quad(\text { by using }(3.13)) \\
= & 2 \int_{a}^{b}\left\{D_{\beta}\left(y(t) D_{\beta} x(t)\right)-D_{\beta}\left(x(t) D_{\beta} x(t)\right)\right\} d_{\beta} t \\
= & 2 \int_{a}^{b} D_{\beta}\left\{(y(t)-x(t)) D_{\beta} x(t)\right\} d_{\beta} t \\
= & 2(y(b)-x(b)) D_{\beta} x(b)-2(y(a)-x(a)) D_{\beta} x(a) .
\end{aligned}
$$

Lemma 3.11 Let $y$ be in the domain of $F$, then for any $c, d \in[a, b], a, b \in I$ such that $a \leq$ $c<d \leq b$, we have

$$
\int_{c}^{d}\left(D_{\beta} y(t)\right)^{2} d_{\beta} t \geq \frac{(y(d)-y(c))^{2}}{d-c} .
$$


Proof Let $x(t)=\frac{y(d)-y(c)}{d-c} t+\frac{d y(c)-c y(d)}{d-c}$. Then $D_{\beta} x(t)=\frac{y(d)-y(c)}{d-c}$ and $D_{\beta}^{2} x(t)=0$. This implies that $x(t)$ is a solution of (3.13) with $f(t)=0$ for all $t \in I$ and

$$
F(y)=\int_{c}^{d}\left(D_{\beta} y(t)\right)^{2} d_{\beta} t
$$

for any $y$ in domain $F$. From Lemma 3.10, we get $F(y)-F(x)-F(y-x)=0$, and consequently $F(y)=F(x)+F(y-x) \geq F(x)$. Therefore,

$$
\begin{aligned}
\int_{c}^{d}\left(D_{\beta} y(t)\right)^{2} d_{\beta} t & \geq \int_{c}^{d}\left(D_{\beta} x(t)\right)^{2} d_{\beta} t \\
& =\int_{c}^{d}\left(\frac{y(d)-y(c)}{d-c}\right)^{2} d_{\beta} t \\
& =\frac{(y(d)-y(c))^{2}}{d-c} .
\end{aligned}
$$

Theorem 3.12 ( $\beta$-Lyapunov inequality) Let $f: I \rightarrow(0, \infty)$ be a continuous function, $s_{0} \in I$. Let $x$ be a nontrivial solution of (3.13) with $x(a)=x(b)=0$, where $a, b \in I$ with $a<b$. Then

$$
\int_{a}^{b} f(t) d_{\beta} t \geq \frac{4}{b-a}
$$

Proof From Lemma 3.10 with $y=0$, we have

$$
F(x)=\int_{a}^{b}\left[\left(D_{\beta} x(t)\right)^{2}-f(t)(x(\beta(t)))^{2}\right] d_{\beta} t=0 .
$$

Let $M=\max \left\{x^{2}(t) ; t \in[a, b]\right\}$ and $c \in[a, b]$ such that $x^{2}(c)=M$. Then $M=x^{2}(c) \geq$ $x^{2}(\beta(t))>0$, and

$$
\begin{aligned}
M \int_{a}^{b} f(t) d_{\beta} & \geq \int_{a}^{b} f(t) x^{2}(\beta(t)) d_{\beta} t \\
& =\int_{a}^{b}\left(D_{\beta} x(t)\right)^{2} d_{\beta} t \\
& =\int_{a}^{c}\left(D_{\beta} x(t)\right)^{2} d_{\beta} t+\int_{c}^{b}\left(D_{\beta} x(t)\right)^{2} d_{\beta} t \\
& \geq \frac{(x(c)-x(a))^{2}}{c-a}+\frac{(x(b)-x(c))^{2}}{b-c} \\
& =M\left[\frac{1}{c-a}+\frac{1}{b-c}\right] \\
& =M\left\{\frac{(b+a-2 c)^{2}}{(c-a)(b-c)(b-a)}+\frac{4}{b-a}\right\} \\
& \geq M \frac{4}{b-a} .
\end{aligned}
$$

Therefore, $\int_{a}^{b} f(t) d_{\beta} t \geq \frac{4}{b-a}$. 


\section{Conclusion and future direction}

This paper was devoted to a presentation of some basic integral inequalities based on our general quantum difference operator, $D_{\beta}$, which is defined by $D_{\beta} f(t)=\frac{f(\beta(t))-f(t)}{\beta(t)-t}, t \neq \beta(t)$, where $\beta$ is a strictly increasing continuous function defined on an interval $I \subseteq \mathbb{R}$ that has one fixed point $s_{0} \in I$. These inequalities are the $\beta$-Hölder, $\beta$-Cauchy-Schwarz, $\beta$ Minkowski, $\beta$-Gronwall, $\beta$-Bernoulli, $\beta$-Lyapunov inequalities, and some related ones. We are looking forward to study in detail the theory of linear $\beta$-difference equations based on $D_{\beta}$. This theory unifies the theory of $q$-difference equations and Hahn difference equations. Also, it includes other types of quantum difference equations.

\section{Competing interests}

The authors declare that they have no competing interests.

\section{Authors' contributions}

All authors contributed equally and significantly in writing this article. All authors read and approved the final manuscript.

\section{Author details}

${ }^{1}$ Department of Mathematics, Faculty of Science, Cairo University, Giza, Egypt. ${ }^{2}$ Department of Mathematics, Faculty of Science, Menoufia University, Shibin El-Kom, Egypt.

Received: 25 June 2014 Accepted: 14 January 2015 Published online: 03 February 2015

\section{References}

1. Annaby, MH, Hamza, AE, Aldowah, KA: Hahn difference operator and associated Jackson-Norlund integerals. J. Optim. Theory Appl. 154, 133-153 (2012)

2. Annaby, MH, Mansour, ZS: q-Fractional Calculus and Equations. Springer, Berlin (2012)

3. Aldowah, KA, Malinowska, AB, Torres, DFM: The power quantum calculus and variational problems. Dyn. Contin. Discrete Impuls. Syst., Ser. B, Appl. Algorithms 19, 93-116 (2012)

4. Birto da Cruz, AMC, Martins, N, Torres, DFM: Symmetric differentiation on time scales. Appl. Math. Lett. 26(2), 264-269 (2013)

5. Kac, V, Cheung, P: Quantum Calculus. Springer, New York (2002)

6. Malinowska, AB, Torres, DFM: The Hahn quantum variational calculus. J. Optim. Theory Appl. 147, $419-442$ (2010)

7. Malinowska, AB, Torres, DFM: Quantum Variational Calculus. Spinger Briefs in Electrical and Computer Engineering-Control, Automation and Robotics. Springer, Berlin (2014)

8. Hahn, W: Über Orthogonalpolynome, die q-differenzengleichungen genügen. Math. Nachr. 2, 4-34 (1949)

9. Aldowah, KA: Generalized Time Scales and the Associated Difference Equations. Ph.D. thesis, Cairo University (2009)

10. Hamza, AE, Ahmed, SM: Existence and uniqueness of solutions of Hahn difference equations. Adv. Differ. Equ. 2013, $316(2013)$

11. Hamza, AE, Ahmed, SM: Theory of linear Hahn difference equations. J. Adv. Math. 4, 2 (2013)

12. Bangerezako, G: An Introduction to q-Difference Equations. Bujumbura (2008)

13. Hamza, AE, Sarhan, AM, Shehata, EM, Aldowah, KA: A General Quantum Difference Calculus (2014, submitted)

14. Malinowska, AB, Martins, N: Generalized transversality conditions for the Hahn quantum variational calculus. Optimization 62(3), 323-344 (2013). doi:10.1080/02331934.2011.579967

15. Agarwal, R, Bohner, M, Petreson, A: Inequalities on time scales: a survey. Math. Inequal. Appl. 4, 535-557 (2001)

16. Bohner, M, Clark, S, Ridenhour, J: Lyapunov inequality for time scales. J. Inequal. Appl. 7(1), 61-77 (2002)

17. Rassias, TM: Survey on Classical Inequalities. Mathematics and Its Applications, vol. 517 (2000)

\section{Submit your manuscript to a SpringerOpen ${ }^{\circ}$ journal and benefit from:}

- Convenient online submission

Rigorous peer review

- Immediate publication on acceptance

- Open access: articles freely available online

- High visibility within the field

- Retaining the copyright to your article 